\title{
Bat Wing Pulmonary Opacities
}

National Cancer Institute

\section{Source}

National Cancer Institute. Bat Wing Pulmonary Opacities. NCI Thesaurus. Code C98272.

A radiologic finding that is detected with frontal chest X-ray or chest CT scan. It is characterized by bilateral perihilar pulmonary shadowing. It is seen in pulmonary edema, pulmonary hemorrhage, pneumonia, carcinoma, and lymphoid malignancies. 